\title{
Prevalence of Anaemia among Rural Women of Eastern Nepal
}

\author{
G M Singh Karki ${ }^{1}$, K. K Karna ${ }^{2}$, A. K. Sinha ${ }^{3}$ \\ ${ }^{1} \mathrm{MD}, \mathrm{MS}$ is in Department of Obs. And Gynecology, Birat Medical College Teaching Hospital, Biratnagar, Tankisinwari-3, Morang, Nepal. \\ ${ }^{2}$ M.Sc. (Biotechnology) is in Department of Biochemistry, Birat Medical College Teaching Hospital, Biratnagar, Tankisinwari-3, Morang, \\ Nepal \\ ${ }^{3} \mathrm{Ph} . \mathrm{D}$ is in Professor, Department of Biochemistry, Birat Medical College Teaching Hospital, Biratnagar, Tankisinwari-3, Morang, Nepal
}

\author{
Corresponding Author \\ Dr. A. K. SINHA*, Ph.D \\ Professor, Department of Biochemistry, Birat Medical College Teaching Hospital \\ Biratnagar, Tankisinwari-3, Morang, Nepal \\ Mobile: 00977-9804043459/9842494062 \\ Email:sinhaamar96@gmail.com \\ Phone No: 0097721-421063, 421182 \\ Fax: 0097721-421063
}

\begin{abstract}
Background: Anaemia is a common health problem among women throughout the world and vast majority of this burden occurs in developing countries. In the present study is to determine the prevalence of anaemia in rural women of Tankisinwari, Eastern Nepal.Method: A cross sectional study was conducted to the non-pregnant women of the age group of 21-50 years during march-August 2015. A total of 735 women were participant in the present study. Cyanmethemoglobin method used for the determination of anaemia. Anaemia was classified as per the World Health Organization (WHO) grading criteria.Results: The overall prevalence of anaemia among rural women was 426(58\%).The majority 291(68.3\%) mild anaemia while moderate anaemia was recorded is $121(28.4 \%)$ and 14(3.3\%) of the anaemic subject had severe anaemia. The mean and standard deviation of age and haemoglobin were $30.72 \pm 8.84$ and $10.23 \pm 1.57$ (g/dl) among anaemic population. Conclusion: The study revealed that anaemia is present at considerable levels among rural women in our study area which provide intensive approaches are required to combat the anaemia in this population.
\end{abstract}

Keywords: Women, Anaemia, Rural area, Prevalence

\section{Introduction}

Haemoglobin is an important respiratory pigment of blood and it performs an important function on Oxygen carrier and it also takes part in Carbon dioxide transport. There are various causes of anaemia, however iron deficiency in the diet is the most common nutritional anaemia in the world and is more common in the rural peoples of poor socioeconomic status.

The WHO global database on anaemia for 1993-2005, covering almost half the world's population, estimated the prevalence of anaemia worldwide at 25 percent ${ }^{(1)}$ Although the prevalence of anaemia is estimated at percent in countries with high development in countries with low development the prevalence is 43 percent. Women are most at risk, with global anaemia prevalence estimates of $42 \%$ in pregnant women and $30 \%$ in non pregnant women aged 15 45 years. ${ }^{(2)}$

India is one of the countries with very high prevalence of anaemia in the world. Nutritional anaemia is a major public health problem in India and is primarily due to iron deficiency .The national Family Health Survey-3(NFHS-3) data suggested that anaemia is widely prevalent among all age groups, and is particularly high nearly $55.3 \%$ amongst the most vulnerable in all women $15-45$ years. ${ }^{(3)}$ It has long been recognized that anaemia is a major public health problem especially among poorer segments of the population in developing countries. ${ }^{(4)}$
Anaemia continues to be a major public health problem Worldwide, particularly among women. ${ }^{(5)}$ It has been estimated that more than one third of the world's women are anaemic; the vast majority of this burden occurs in developing countries. ${ }^{(6-7)}$ It is a common health problem among the women of $18-45$ years age. ${ }^{(7-8)}$ Due to different socioeconomic and other influencing factors, the epidemiology of anaemia varies among different regions. ${ }^{(5)}$ Present study was conducted to highlight this situation in women age 20-50 years of rural areas, Tankisinwari of Eastern Nepal.

Iron deficiency anaemia is the most common form of malnutrition in the world and is the eight leading cause of disease in girls and women in developing countries. ${ }^{(9)}$ Its estimated prevalence in South East Asia 50-70\%..$^{(10-11)}$ In another study, Iron deficiency and anaemia were also most prevalent among women and young children with the highest prevalence in low income countries. ${ }^{(12)}$

The determination of factors that influence the occurrence of anaemia in a population is fundamental for the implementation of control measures. In view of this, our aim is to determine the prevalence of anaemia among ever married women of from the Tankisinwari, village development committee (VDC) of Eastern Nepal and to explore some factors commonly associated with anaemia. 


\section{International Journal of Science and Research (IJSR)}

ISSN (Online): 2319-7064

Index Copernicus Value (2013): 6.14 | Impact Factor (2014): 5.611

\section{Material and Method}

This is community based cross sectional study conducted in rural area of Tankisinwari VDC, a field practice area of Department of Community Medicine, Birat Medical College, Morang district of Eastern Nepal during MarchAugust 2015.The data were assessed by the questionnaire with the help of Medical students of Birat Medical college. Sample size 735 women from the Tankisinwari VDC, Morang district of eastern Nepal selected for the study. Present study was conducted to highlight this situation in women aged 20-50 years of rural areas, Tankisinwari VDC, of Eastern Nepal.

Women who were pregnant at the time of survey were not included in the study. Ages in years were obtained from the date of birth which the majority of the women could recall.

The blood haemoglobin concentrations of the subjects were carried out by Cyanmethemoglobin method ${ }^{(13)}$ (Drebkin et al 1932) which was checked each day at Birat Medical College, Tankisinwari Hospital Laboratory.

The classification of anaemia was based upon criteria developed by the WHO ${ }^{(6)}$. Mild anaemia was defined as haemoglobin concentration lay between 10.0-11.9 g/dl, moderate anaemia was defined as haemoglobin concentration lay between $7.0-9.9 \mathrm{~g} / \mathrm{dl}$ and severe anaemia was defined as haemoglobin concentration $<7 \mathrm{~g} / \mathrm{dl}$.

Statistical package for social science (SPSS 20.0) was used data analysis. Data were presented as mean \pm SD. Statistical significance was calculated using chi-square test and $p$ value $<0.05$ was considered significant.

The subject protocol was approved by ethics community of BMC. The study protocol was fully explained to the subjects and consent was obtained prior the commencement of the study.

\section{Results}

The total sample by the survey was 735 women aged between 21-50 years. Most of the women were between 2125 years of age $(43.3 \%)$ followed by $41-45$ years $(13.3 \%), 36$ 40 years $(13.1 \%)$ while lower number of subject accounted for 26-30 years age group.

There was significance difference in distribution of study population with their age group since the $\mathrm{p}$ value is 0.000 .

Table 1: Characteristics of study population $(\mathrm{N}=735)$

\begin{tabular}{|c|c|c|}
\hline Age Group & Number & Percentage (\%) \\
\hline $20-25$ & 318 & $43.3 \%$ \\
\hline $26-30$ & 93 & $12.6 \%$ \\
\hline $31-35$ & 86 & $11.7 \%$ \\
\hline $36-40$ & 96 & $13.1 \%$ \\
\hline $41-45$ & 98 & $13.3 \%$ \\
\hline $46-50$ & 44 & $6.0 \%$ \\
\hline Chi-Square $=$ & 390.918 & P value $=0.000$ \\
\hline
\end{tabular}

The severity of anaemia according to the age group is shown in table 2. The over all prevalence of anaemia defined on a haemoglobin $<11.9 \mathrm{gm} / \mathrm{dl}$ was $58 \%(426 / 735)$. The majority $(68.3 \%)$ had mild anaemia only $28.4 \%$ were moderate anaemia and $3.3 \%$ were severe range of anaemia. The distribution of age and grading of severity of anaemic mild, moderate and severe was significant/non significant since the $p$ value was 0.356 . When we see the association between grades of anaemia with attributes, we find that age category is also showing significant/nonsignificant association with grades of anaemia.

Table 2: Age group wise prevalence of anaemia among rural women population

\begin{tabular}{|c|c|c|c|c|c|}
\hline \multirow{2}{*}{$\begin{array}{c}\text { Age Group } \\
\text { (In years) }\end{array}$} & MILD & MODERATE & SEVERE & TOTAL & NORMAL \\
\cline { 2 - 5 } & $131(45.1 \%)$ & $52(43.0 \%)$ & $4(28.6 \%)$ & $187(43.9 \%)$ & $131(42.4 \%)$ \\
\hline $20-25$ & $34(11.7 \%)$ & $26(21.5 \%)$ & $2(14.3 \%)$ & $62(14.6 \%)$ & $31(10.0 \%)$ \\
\hline $26-30$ & $34(11.7 \%)$ & $12(9.9 \%)$ & $3(21.4 \%)$ & $49(11.5 \%)$ & $37(12.0 \%)$ \\
\hline $31-35$ & $38(13.0 \%)$ & $7(5.8 \%)$ & $4(28.6 \%)$ & $49(11.5 \%)$ & $47(15.2 \%)$ \\
\hline $36-40$ & $38(13.0 \%)$ & $18(14.8 \%)$ & - & $56(13.1 \%)$ & $42(13.6 \%)$ \\
\hline $41-45$ & $16(5.5)$ & $6(5.0 \%)$ & $1(7.1 \%)$ & $23(5.4 \%)$ & $21(6.8 \%)$ \\
\hline $46-50$ & $291(68.3 \%)$ & $121(28.4 \%)$ & $14(3.3 \%)$ & $426(58.0 \%)$ & $309(42.0 \%)$ \\
\hline Overall & \multicolumn{3}{|c|}{ P Value } & \multicolumn{2}{c}{0.356} \\
\hline
\end{tabular}

The mean and standard deviation of age and haemoglobin were $30.72 \pm 8.84$ and $10.23 \pm 1.57(\mathrm{~g} / \mathrm{dl})$ among anaemic population.

Table 3: Comparison of Mean and SD of age and haemoglobin concentration

\begin{tabular}{|c|c|c|c|}
\hline \multicolumn{2}{|c|}{ Subjects } & Mean & SD \\
\hline Anemic & Age & 30.72 & 8.845 \\
\hline Non anemic & Age & 31.50 & 9.067 \\
\hline Anemic & $\mathrm{Hb}$ & 10.23 & 1.572 \\
\hline Non anemic & $\mathrm{Hb}$ & 13.15 & 0.992 \\
\hline
\end{tabular}

\section{Discussion}

Nutritional anaemia is a common all over and there are around billion iron deficient people in the world ${ }^{(14)}$.There are very few studies on the prevalence of anaemia among rural women in comparison to other groups like adolescent and children in the world and it is the case in Nepal too.

Several studies have also found a negative association between the socioeconomic situation (SES) and anaemia prevalence. ${ }^{(15-17)}$ Women from poorer households are usually found to have higher anaemia prevalence. A poor socioeconomic situation (SES) is known to be associated 


\section{International Journal of Science and Research (IJSR) \\ ISSN (Online): 2319-7064}

Index Copernicus Value (2013): 6.14 | Impact Factor (2014): 5.611

with a number of factors, such as high parity, short birth interval, poor diet both in quantity and quality, lack of health and nutrition awareness and high rate of infectious diseases and parasitic infections. Since the socioeconomic situation (SES) is an important determinant of access to health care, poor people have often limited to medical attention and preventive measures ${ }^{(18)}$ increasing their risk of becoming anaemic.

In India, the prevalence of anaemia among all ages remains very high. The prevalence rate among rural pregnant women is $84.9 \%$ with $9.9 \%$ having severe anaemia. ${ }^{(19)}$ Another study among ${ }^{(20)}$ rural women population the prevalence is $70.1 \%$.Another study done in Morang district of Nepal and south western region of Nepal in reproductive age group the prevalence of anaemia was $67.3 \%$ and $54.89 \%$ respectively. ${ }^{(21-22)}$

There are many studies on anaemia in pregnancy in Nepal, the prevalence of anaemia was $62.2 \%$ out of which $3.6 \%$ with severe anaemia showed in a study done in Kathmandu. (23) Another study showed that the prevalence of anaemia was $47.2 \%$ of Morang district of Nepal. ${ }^{(24)}$ Similarly high prevalence $(50-60 \%)$ of anaemia was noted in the study carried out for Nepal in $1988 .^{(25)}$

According to the present study, showed that anaemia of moderate severity among studied women was considered a health problem. Studied on prevalence of anaemia from different states of rural India, reported a prevalence of anaemia from $46-90 \%{ }^{(26-28)}$

In females, the higher prevalence of anaemia observed reflect the adverse effect of lower dietary iron intake with menstrual blood loss, which imposes extra demand for iron. Higher prevalence of anaemia now found at the age of 21-25 years. This is in agreement with many studies reported elsewhere. ${ }^{(29-30)}$

In the recent study, conducted in Morang district of Nepal, the prevalence of anaemia in adolescent age group both sexes i.e. males and females were found $47.7 \%$ and $52.3 \%$ respectively. ${ }^{(31)}$ Our study further revealed that anaemia was mostly recorded at the age of 21-25 years. In many studies was found that anaemia is a common problem in women due to low income they are unable to take dietary food, lack of awareness is also main cause of anaemia.

\section{Conclusion}

The initial use for this indicator is to identify women with anaemia who require iron supplementation and complementary care. It can be use on a proxy for general nutritional status. Population based assessments can estimate prevalence of anaemia and iron deficiency in communities and regions; identify high risk population for intervention; monitor prevention and treatment programs; and identify need and advocate for food fortification and iron supplementation programs. For surveillance purposes, rapid assessments of anaemia are conducted each year and population level surveys every five year.

\section{Acknowledgement}

The authors would like to thanks the medical students and department of Community Medicine, BMC for helping the collection of data. Authors are also thankful to all participants and community people for their cooperation in this study.

\section{References}

[1] Source: de Benoist B et al., eds. worldwide prevalence of anaemia 1993-2005. WHO Global Database on Anaemia Geneva,World Health Organization, 2008.

[2] McLean E, Cogswell M, Egli I et al. Worldwide prevalence of anaemia, WHO Vitamin and Mineral nutrition Information System, 1993-2005.

[3] National Nutrition Monitoring Bureau, Diet and Nutritional Status of rural population and prevalence of hypertension among adults in Rural Areas,NMBS Technical Report No.24,2006,NNMB,NIN,Hyderabad.

[4] Centers for Disease Control and Prevention. Recommendation to prevent and control iron deficiency in the United States.Morb Mortal Wkly Rep 1998; 47(RR-3):1-36.

[5] Bentley ME, Griffiths PL. The burden of anemia among women in India. European J Clinical Nutr 2003; 57:5260.

[6] World Health Organization: The prevalence of anaemia in women: a tabulation of available information. WHO, Geneva, Switzerland.1992.

[7] Dangour AD, Hill HL, Ismail SJ. Haemoglobin status of adult non-pregnant Kazakh women living in Kzylorda region,Kazakhstan. European J Clinical Nutr 2001;55:1068-75.

[8] Isah HS, Fleming AF, Ujah IAO, et al. Anaemia and iron status of pregnant and non pregnant women in the Guinea Savana of Nigeria.Ann Trop med Parasitol 1985;79:485-92.

[9] World Bank. World development report investing in health. New York: Oxford University Press, 1993.

[10] Garcia M, Mason J.Second report of the world nutrition situation. Geneva: United Nations Adminstrative Committee on Co-ordination/sub-committee on Nutrition, 1992.

[11] Mother care. Prevalence of maternal anaemia in developing countries. Working paper No.7.Arlington, Va, USA: John Snow 1992.

[12]De Maeyer EM. Preventing and controlling Iron Deficiency Anaemia through primary Health Care. A guide for Health Administrator and Programme Planners. Geneva: World Health Organization, 1989.

[13] Drabkin D.L., Austin J.M.,1932. Spectrophotometric constants for common haemoglobin derivatives in human,dog and rabbit blood. Journal of biological Chemistry., 1932;98:719-33.

[14]De Maeyer E, Adiels. Tegman M.The prevalence of anaemia in the world. World Health Stat Quarter 1985; 38:302-16.

[15] Carlo EB, Sosa EM. Iron status in non-pregnant women of child bearing age living greater Buenos Aires. Eur.J Clin Nutr., 1991;45:215-20.

[16] Isah HS, Fleming AF, Ujah IAO, EKwempu C.C. Anaemia and iron status of pregnant and non-pregnant

\section{Volume 4 Issue 12, December 2015}




\section{International Journal of Science and Research (IJSR) \\ ISSN (Online): 2319-7064}

Index Copernicus Value (2013): 6.14 | Impact Factor (2014): 5.611

women in the Guinea savanna of Nigeria. Ann Trop Med Parasitol.,1985;79:485-93.

[17] Johnson A, Latham MC, Roe DA. The prevalence and etiology of the neutritional anaemias in Guyana.Am J Clin Nutr., 1982;35:309-18.

[18]Lovell C, Abed FH. Scaling up in health: two decades of learning in Bangladesh. In Rohde J, Chatterjee M, Morley D, eds. Raching health for all. Bombay: Oxford University Press., 1988;212-32.

[19] Teoteja G, Singh P. Micronutrient deficiency disorder in 16 districts of India. Report of an ICMR Task Force Study-District Nutrition Project. Part., 2001;1.

[20] Maiti S, Ali KM, Ghosh A, Ghosh K, Ghosh D, Paul S. Prevalence of Anaemia among Rural Women Population of Paschim Medinipur,West Bangal,india. Nepal Journal of Medical Sciences 2013;2(1):9-12.

[21] A.K. Sinha ${ }^{* 1}$, G.M. Singh Karki ${ }^{2}$, S.J. Walawalkar ${ }^{3}$, N. Manandhar ${ }^{4}$, K. K. Karna ${ }^{4}$ : Prevalance of Anemia amongst Women in Reproductive Age Group of Morang District, Nepal. Journal of Biomedical and Pharmaceutical Research (JBPR) 2 (1) 2013, 48-51

[22] A.K.Sinha ${ }^{*}$, R.K. Dubey, P.Padmavathi, Y.R.Neupane, J.Archana, N.Gautam. Prevalence of Anaemia amongst Reproductive Age Group Women of South Western Nepal. The Journal of Obstrtrics \& Gynecology and Reproductive Biology. Photon. 2013; 114:170-174.

[23] Bondevik GT, Ulstein M, Lie RT, Rana G, K vale G. The prevalence of anaemia in pregnant Nepali women-a study in Kathmandu. Acta Obstet Gynecol Scand., 2000; 79: 341-9.

[24]A.K Sinha, G.M. Singh Karki, Sanjay Yadav and Md.Nazrul Islam. Prevalence of anaemia during pregnancy in the women of Eastern Nepal: IJPBA., 2012;3(5):1024-1026.

[25] Ulstein M, Rana G, Yangzom K et al. Some fetal and pregnancy parameters in Nepal. Acta Obstet Gynecol Scand., 1988; 67:47-52.

[26] Kanani S.Combating anemia in adolescent girls: a report from India. Mothers Child 1994; 13:1-3.

[27] Pathak P, Singh P, Kapil U, Raghuvanshi RS. Prevalance of iron,Vitamin A and iodine deficiencies among adolescent pregnant mothers.Indian J Pediatr 2003;70:299-301.

[28] Sampath KV, Rajaratnam A. Prevalaence of anemia and hookworm infestation among adolescent girls in one rural block of Tamilnadu.Indian J Metern Child Health 1997;8:73-75.

[29] Powell EDV. Levels of haemoglobin in an adolescent population in Dublin.J Ir Med Assoc 1960;46:157-161.

[30] Expert Scientific Working Group. Summary of a report on assessment of the iron nutritional status of the United States population. Am J Clin Nutr 1985;42:1318-30.

[31] Sinha AK, Singh GMK, Karna KK.2012. Prevalance of anaemia amongst adolescent in Biratnagar,Morang Dist. Nepal. IJPBA 2012; 3(5):1077-81.

\section{Author Profile}

Dr. G. M. Singh Karki, MD, MS is in Department of Obs. And Gynecology, Birat Medical College Teaching Hospital, Biratnagar, Tankisinwari-3, Morang, Nepal.

K. K. Karna, M. Sc. (Biotechnology) is in Department of Biochemistry, Birat Medical College Teaching Hospital, Biratnagar, Tankisinwari-3, Morang, Nepal

Dr. A. K. Sinha, Ph. D is in Professor, Department of Biochemistry, Birat Medical College Teaching Hospital, Biratnagar, Tankisinwari-3, Morang, Nepal 